\title{
Knowledge among the rural parents about the vaccinations and vaccination coverage of children in the first year of life in Papua New Guinea - analysis of data provided by Christian health services
}

\author{
Ewelina Gowin ${ }^{1 *}$ (D) Jerzy Kuzma ${ }^{2}$ and Danuta Januszkiewicz-Lewandowska ${ }^{1}$
}

\begin{abstract}
Knowledge among the rural parents about the vaccinations and vaccination coverage of children in the first year of life in Papua New Guinea - analysis of data provided by Christian Health Services.

Background: This analysis aimed to assess rural parents' knowledge about the diseases prevented by vaccinations and establish vaccination coverage in PNG.

Methods: Knowledge of vaccinations was checked through a standard questionnaire (five closed questions). We analyzed data on vaccination coverage from 2016 to 2018 from all Catholic health facilities. Analyzed vaccinations were the pentavalent vaccine (DTaP-HiB-HepB) and measles vaccine given in the first year of life. Coverage was calculated based on the number of vaccines used compared to the number of eligible children. Analyzed vaccinations were the pentavalent vaccine (DTaP-HiB-HepB) and measles vaccine given in the first year of life.

Results: Fifty-six parents, including 52 mothers and four fathers, participated in the interview. Many parents (46\%) understood that the vaccine prevents diseases. During the analyzed period, 25,502 doses of measles vaccine were given, 31,428 children were vaccinated with the pentavalent vaccine. In 2016, the measles vaccine coverage rate was 26.6 and 33.4\% for the pentavalent vaccine. In 2017, measles and pentavalent vaccines' coverage rate was 12.5 and $16.6 \%$, respectively. There were significant differences in immunization coverage between provinces. A decreasing trend in the number of administered vaccinations was observed.

Conclusion: The results of this analysis demonstrate that in PNG, the majority of children are not fully immunized. There are significant differences in the vaccination coverage between provinces. As protection from diseases is low, there is a very high risk of an outbreak of the vaccine-preventable disease in the community.

Delivery of vaccinations in PNG encounters many barriers, from access to healthcare services to natural disasters and inter-tribial conflicts.
\end{abstract}

Keywords: Vaccinations, Rural parents, The coverage rate

\footnotetext{
*Correspondence: ewego@poczta.onet.pl

${ }^{1}$ Health Promotion Department, Poznan University of Medical Sciences, Fredry 10, 61-701 Poznan, Poland

Full list of author information is available at the end of the article
} 


\section{Background}

Papua New Guinea (PNG) has a population of around 8 million people, with a birth cohort estimated at 200,000 children $[1,2]$. This is a country where vaccinepreventable diseases are still a problem. In 2017, according to Annual Report on Child Morbidity and Mortality in PNG, there were 11 cases of tetanus (four deaths), 25 cases of whooping cough, 28 cases of acute flaccid paralysis (three deaths) and three cases of measles [3]. Recent cases of polio just reminded us of this and drew attention to that unresolved problem.

In PNG, the immunization program was launched in 1977, providing vaccinations against tuberculosis, polio, diphtheria, pertussis, and tetanus. In 1981, the Expanded Program on Immunization (EPI) was started. In 2009, the pentavalent vaccine replaced the tetravalent vaccine used in 2007 and 2008. The pneumococcal vaccine was introduced in 2013. The GAVI supports the introduction of the new vaccines. Vaccinations supported in PNG by GAVI are measles, polio, pneumococcal and pentavalent vaccines [4].

The Family Health Unit organizes immunization services in PNG in the Health Improvement Branch of the National Department of Health. They are offered as a part of public health services through 800 Maternal and Child Health $(\mathrm{MCH})$ clinics. Provincial Cold Chain Logistics Officers (PCCLO) are responsible for managing vaccines at a regional level with support from the Provincial Family Health Coordinator. At the district level, EPI is managed by the District Manager through the health facility (Maternal \& Child clinics and Well Baby clinics) Sister-In-Charge. It covers $30 \%$ of children; the rest of the children are reached through outreach services. There are 29 outreach clinics for every 1000 children under the age of 5 years [2]. Approximately $63 \%$ of PNG health facilities are government-owned, and religious organizations organize the remaining. Church organizations offer a significant proportion of immunization services, and $99 \%$ of the population declares the Christian religion [1,2]. The people targeted by EPI include those in the first year of life, children entering and leaving school (age 6 and 13 years), and pregnant women. The vaccination schedule in PNG is presented in Table 1.

The National Health Information System does monitoring of vaccination. It is difficult to establish actual vaccination coverage due to the absence of a recent coverage survey. There are different types of data provided by the government (Sector Performance Annual Review), the World Health Organization (WHO), and UNICEF $[5,6]$.

Available data from the National Health Information System, WHO, and UNICEF estimates are presented in Table 2. Differences between data make it very difficult to analyze it and share it with the provinces. All reports indicate a decrease in vaccination coverage, with a simultaneous dynamic increase in the number of inhabitants in the last 5 years [5-7]. The aim of this analysis was to assess knowledge among rural parents about the diseases prevented by vaccinations and to establish vaccination coverage in PNG.

\section{Material and methods}

It was a cross-sectional study performed by a healthcare worker in villages in 2018/2019. One healthcare worker collected data during a routine visit in villages in Simbu Province (Kervagi district) and Morobe Province (Finschhafen district). During visits in every household, a healthcare worker identified all children younger than 5 years. Parents of those children were the potential participants. To be eligible for recruitment into the survey, the caregivers were required to consent, live in Simbu or Morobe Province, and have children who were younger than 5 years. Only families who agreed to participate in the study were asked about vaccination status.

Knowledge on vaccinations was checked through an interviewer-administered questionnaire. The instrument collected socio-demographic information of caregivers, knowledge of immunization, knowledge about vaccinepreventable diseases, history of vaccines received by the child. Due to a high illiteracy rate, data were collected by face-to-face, interview-based questionnaire. Most of the

Table 1 Vaccination schedule in PNG

\begin{tabular}{|c|c|}
\hline Vaccine & Age at administration \\
\hline BCG (Bacillus Calmette-Guérin, vaccine against tuberculosis) & birth \\
\hline HepB (vaccine against hepatitis B) & birth \\
\hline DTP Hib HepB (vaccine against diphtheria, tetanus, pertussis, Haemophilus influenzae type B, and hepatitis B) & 1, 2, 3 months \\
\hline IPV (inactivated poliovirus given by injection) & 3 months \\
\hline MR (vaccine against measles and rubella) & $6,9,18$ months \\
\hline OPV (weakened poliovirus given by mouth) & $1,2,3$ months \\
\hline Pneumococcal (vaccine against Streptococcus pneumoniae) & $1,2,3$ months \\
\hline TT (Tetanus toxoid vaccination) & 7,13 years \\
\hline
\end{tabular}


Table 2 Vaccination coverage in PNG in years 2014-2017

\begin{tabular}{llllll}
\hline Vaccine & Data source & $\mathbf{2 0 1 4}$ & $\mathbf{2 0 1 5}$ & $\mathbf{2 0 1 6}$ & $\mathbf{2 0 1 7}$ \\
\hline DTP & National data & $61 \%$ & $54 \%$ & $44 \%$ & $51 \%$ \\
& WHO/UNICEF estimates & $73 \%$ & $73 \%$ & $72 \%$ & $62 \%$ \\
\multirow{4}{*}{ Measles } & Our Survey & - & - & $33.4 \%$ & $16.6 \%$ \\
& National data & $65 \%$ & $60 \%$ & $51 \%$ & $43 \%$ \\
& WHO/UNICEF estimates & $84 \%$ & $79 \%$ & $70 \%$ & $62 \%$ \\
& Our survey & - & - & $26.6 \%$ & $12.5 \%$ \\
\hline
\end{tabular}

questions were close-ended. The questionnaire was available in two languages English and regional language, TOKPISIN. The questionnaire was constructed for this study based on available literature on attitudes to vaccinations, and has not been published. It was validated by a group of students of the Divine Word University.

Vaccination status was checked based on vaccination records or checking the presence of a BCG scar. Parents were asked to bring a vaccination booklet, if available. Each child's vaccination record was checked against the recommended EPI immunization schedule.

The surveyor received 1 day of training, covering an overview of research methods, interview strategies, and ethical considerations. Data obtained from questionnaires were uploaded to excel forms (no personal data were introduced to the system). The questionnaire is included as a supplementary file.

Analysis of vaccination coverage was based on the data provided by CHS from 2016 to 2018. Each year the population of children younger than 1 year covered by CHS was around 50,000, which is $1 / 4$ of the birth cohort. The target population was based on data from the Census. The CHS of PNG is the organization that represents all Christian Churches that provide health care service throughout PNG. CHS gets its funding from the Government of PNG through the National Department of Health. CHS is responsible for managing all 29 churchrun health agencies within PNG. There are 713 healthcare facilities (hospitals, urban clinics, health centers, aid posts) in 22 provinces. We analyzed data on vaccination coverage from 2016 to 2018 from all Catholic health facilities that provide vaccinations in all PNG provinces. Analyzed vaccinations were the pentavalent vaccine (DTaP-HiB-HepB) and measles vaccine given in the first year of life.

\section{Patient and public involvement}

The researchers did not assume any significant risk to themselves and the participants; the data are not of a sensitive category. We adopt an implied consent, which assumes that when, following the study's information, the participant agrees to self-administer the questionnaire or participate in the interview, he/she agrees to take part in the survey. An informed verbal consent was obtained from all participants. Being aware of the high illiteracy, the ethics committee approved this procedure. The study received the approval of the ethics committee of the Divine Word University.

\section{Results}

A total of 58 caregivers were eligible for the study; 56 parents, including 52 mothers and four fathers from two areas: Simbu Province (Kervagi district) and Morobe Province (Finschhafen district), participated in the interview. The response rate was $96.55 \%$. The parents' education level was very low: six had never been to school, 17 was four o less grade, 29 have 5-8 grade, seven have 9 or 10 classes completed. The average number of children in families was 2.5 children. The age range for participating children varies between 9 months and 9 years, while participating children's average age was 3.3 years. Thirty-eight out of 56 parents (68\%) have no vaccination records in the child's health book.

Many parents (26 out of $56 ; 46 \%$ ) understood that the vaccine prevents diseases. Other single opinions were that "vaccination can prevent a child for a disability" and "help a child to grow well" Almost all the women (50 out of 56) admitted to having no idea how a vaccination works. Only a few women mentioned that vaccination: "allow a child to grow" or "strengthen the child." Seventy percent thought that the reason for vaccination is to prevent the sick (40 out of 56) or even death. To persuade other parents to vaccinate their child, the highest proportion (38\%, 21 out of 56) will inform that vaccination "prevent a child from becoming sick" or from death. Others will provide a more general statement that vaccinated children "grow well." Knowledge of diseases that can be prevented by vaccinations was shallow. The most frequent known disease was polio 18 people, and tuberculosis (TB) 15. Other conditions were tetanus and hepatitis B mentioned by four parents. The average number of known diseases preventable by vaccination was 0.8 (details in Tables 3 and 4).

During the analyzed period, 31,428 children were vaccinated with the pentavalent vaccine, and 25,502 doses of measles vaccine were given. In 2016, the measles vaccine coverage rate was 26.6 and $33.4 \%$ for the pentavalent vaccine. In 2017, measles and pentavalent vaccines' coverage rate was 12.5 and $16.6 \%$, respectively. There were significant differences in immunization coverage between provinces (Fig. 1). In 2016, greater than $80 \%$ coverage was noted in one province for the measles vaccine and four provinces for the pentavalent vaccine. In 2017, in none of the provinces, the coverage rate, neither for pentavalent nor for measles vaccine, was higher than 


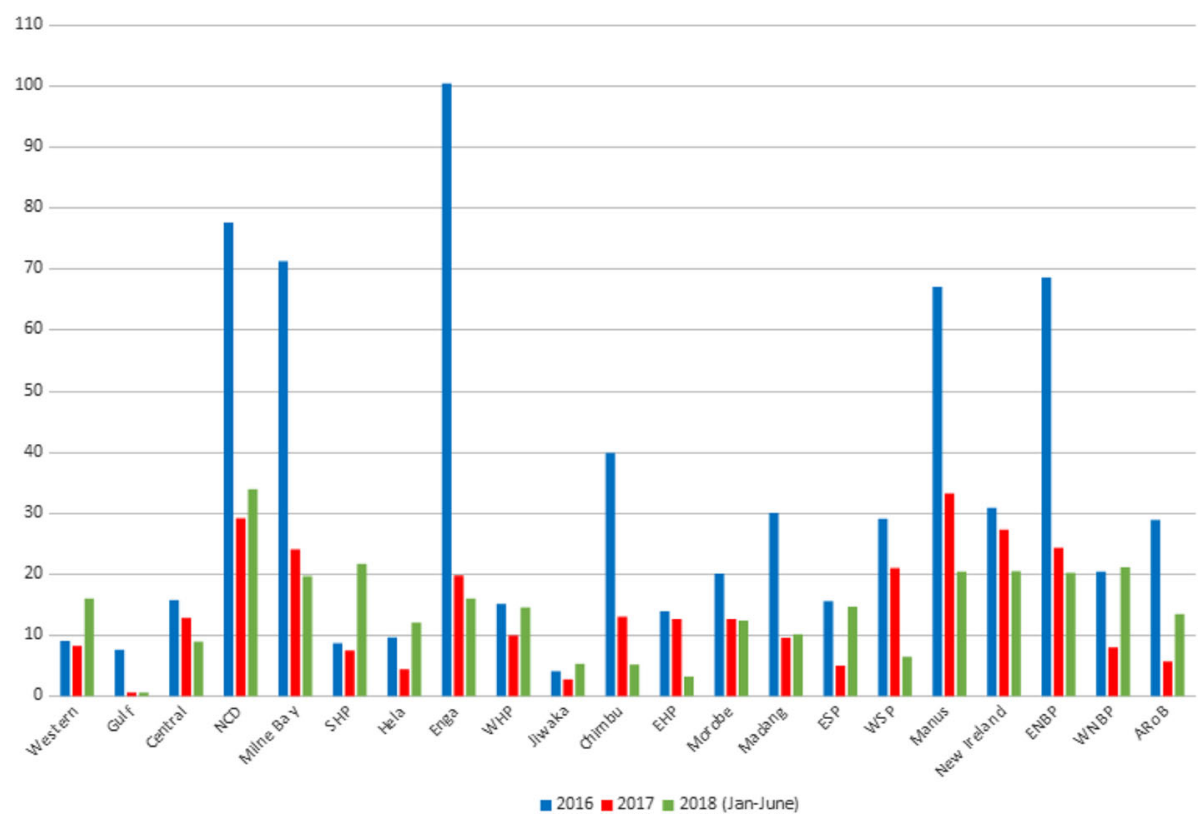

Fig. 1 Vaccination service utilization in the clinics

$80 \%$ (Fig. 2). A decreasing trend in the number of administered vaccinations was observed.

Eighteen children had a vaccination booklet; half of them had one vaccination visit. The commonest given vaccine was DTP (diptheria, tetanus, pertussis), followed by measles and rubella vaccines given in 16 patients. Details are presented in Table 3.

\section{Discussion}

Our analysis showed large differences between vaccination coverage in each province. In some medical facilities, the vaccination coverage was even more than $100 \%$. This does not mean that some children were vaccinated twice, but that there were more children than before. It was a case in 10 health facilities in three provinces. The lowest coverage was in rural regions. At least

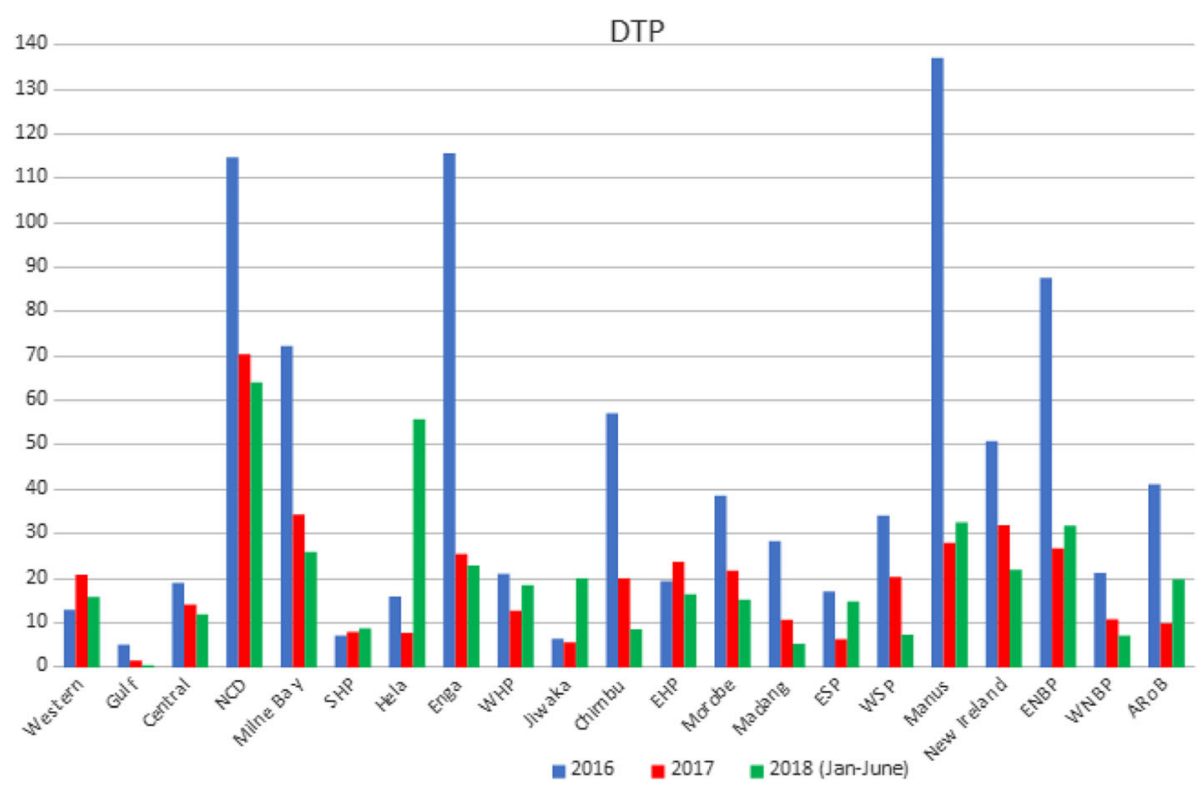

Fig. 2 Percentage of three doses of pentavalent and measles coverage in children under 1 year of age in all provinces of PNG 
Table 3 Results of the parents survey

\begin{tabular}{l} 
Number of parents $(\boldsymbol{n}=\mathbf{5 6})$ \\
List, what diseases can be prevented by vaccination? \\
Polio \\
TB \\
tetanus \\
hepatitis B \\
pneumonia \\
What is the role of vaccination for the child? \\
"the vaccine prevents the child from the sick" \\
"vaccination can prevent a child from disability" \\
"help a child to grow well" \\
Any idea how vaccination is working? \\
no idea \\
"allow a child to grow" \\
"strengthen the child" \\
Why it is important to vaccinate your child? \\
"to prevent the sick" \\
"to prevent the death" \\
What would you tell if you like convince your neighbor to take child for \\
vaccination? \\
"vaccination prevent a child from becoming sick" \\
"vaccination prevent a child from death" \\
"don't know" \\
\hline
\end{tabular}

$60 \%$ of the population lives in areas not accessible by road. Access to services can be, in these provinces, the biggest problem in vaccine delivery. The vaccination coverage in only one province (Morobe) is equal to median vaccination coverage in PNG. Moreover, one province (Chibu) within the analyzed period experienced a marked decline in vaccination rates - to rates lower comparing to the country average.

In PNG, there is often only one static clinic per week at the health center level, resulting in long waiting times for services. Although vaccinations are free of charge, some health facilities require user fees to run the operational costs. This discourages attendance at clinics unless the child is sick. It is proved by a mean number of outpatient visits per person per year, which in PNG is 1.28 [7]. Neonatal mortality and under 5-year mortality are among the highest in this region of the world (57/ 1000 live births) [1, 2, 7]. Services provided by the healthcare facilities also experience many troubles: lack of vaccinations, $30 \%$ of healthcare facilities are experiencing problems with vaccinations supply or issues with maintaining cold chain [7]. The other problem is a suboptimal number of healthcare professionals. According to the official data in PNG, there are 32 pediatricians, 0.5 physicians per 10,000 population, and 5.3 nurses per 10,000 people [2].

PNG has a relatively low coverage of essential services (Universal Health Coverage) according to WHO [6]. Antenatal care is an indicator of access to and use of health care during pregnancy, and its low use is one of the well-known risk factors for incomplete vaccination. Mean antenatal care use in PNG is estimated at 54\% [8]. It varies between provinces, from $30 \%$ in Jiwaka to $98 \%$ in NCD [7]. In regions with the lowest use of antenatal care, CHS's vaccination coverage was also low. In a study conducted by Russo in Cameroon, children born at health facilities had a higher immunization coverage rate than those born at home [9].

Table 4 Vaccination status based on child's book and observation from the rural setting of Papua New Guinea, 2019 ( $n=18)$

\begin{tabular}{|c|c|c|}
\hline & Number of patients $(n=18)$ & Percentages (\%) \\
\hline One vaccination visit & 9 & $50.00 \%$ \\
\hline Two vaccination visits & 3 & $16.67 \%$ \\
\hline Three vaccination visits & 6 & $33.3 \%$ \\
\hline Measles vaccine & $\begin{array}{l}16 \\
\text { ( } 8 \text { 1dose; } 6-2 \text { doses, } 2-3 \text { doses) }\end{array}$ & $88.89 \%$ \\
\hline Mumps vaccine & 0 & \\
\hline Rubella vaccine & $\begin{array}{l}16 \\
\text { (8 1dose; 6-2 doses, 2-3 doses) }\end{array}$ & $88.89 \%$ \\
\hline Varicella vaccination status & 0 & \\
\hline Te De Pe (DTP) vaccinated & 17 & $94.44 \%$ \\
\hline Hepatitis $B$ vaccination $x 1$ dose & 16 & $88.89 \%$ \\
\hline Hepatitis B vaccination $\times 2$ doses & 5 & $27.78 \%$ \\
\hline Hepatitis B vaccination $\times 3$ doses & 1 & $5.55 \%$ \\
\hline Polio Oral vaccination $\times 1$ dose & 15 & $83.33 \%$ \\
\hline Polio Oral vaccination $\times 2$ doses & 7 & $38.89 \%$ \\
\hline Polio Oral vaccination $\times 3$ doses & 6 & $33.3 \%$ \\
\hline BCG scar present & 18 & $100.00 \%$ \\
\hline
\end{tabular}


The percentage of supervised deliveries in PNG is estimated at $37 \%$ [7].

The other problem is the lack of public understanding of the need for vaccinations $[10,11]$. There are considerable difficulties in communication. The adult literacy rate is estimated at $63.4 \%$ [1]. So far, no opposing opinions about vaccinations have been noticed in PNG, but the understanding of an idea of vaccinations is poor. For some people, there is no difference between vaccination - prevention and treatment. They view injection as a treatment. The expected benefit of participation in the survey can be raising awareness of vaccinations and their importance in protecting children and adults.

Natural disasters and military conflicts also cause difficulties in access to health services.

On 26 February 2018, the earthquake took place in four provinces Hela, Southern Highlands, Western Province, and Enga. Five hundred fourty-four thousand people were affected (46\% children; 17,419 children in age 0-12 months). This natural disaster was then followed by inter-communal fighting in Hela Province.

This also caused considerable problems in vaccination. Out of 86 health facilities, 18 were severely damaged. UNICEF estimated that only $10 \%$ of the target population (children younger than 5 years) received pentavalent and measles, rubella vaccination in this province [12].

According to the National Health Information System, the measles vaccine coverage in 2016 was $51 \%$ [7]. None of the provinces reported over the target $80 \%$. The proportion of districts reporting less than 50\% DTP3 (all three doses of vaccine) coverage was as high as $60 \%$. Only $8 \%$ of provinces reported vaccination coverage greater or equal to $90 \%$.

Data concerning the vaccine coverage obtained from CHS are much lower than the official ones. Data recording and reporting in health facilities was a shortcoming identified earlier by researchers performing studies in PNG [13-15]. Wiesen, in 2014 in a study on assessing the hepatitis B birth dose vaccination program in PNG, found out that only $17 \%$ of the health facilities were able to provide a vaccination coverage figure [13].

A lack of reliable demographic data also causes the problem with an estimation of the vaccination coverage. In PNG, birth and death registration systems are not yet sufficiently developed to accurately estimate a birth cohort. The population is growing very fast; hence, an entire birth cohort seems to be more significant. The latest polio epidemic showed that the pediatric population might be more significant than assumed. In the first round of catch-up vaccination action covering the three high-risk provinces of Morobe, Madang, and the Eastern Highlands, the estimated number of children was 289, 582 , but 303,907 (105\%) children under 5 years old were vaccinated $[16,17]$
A field survey is another way to obtain vaccination coverage. It can be done by checking patients' vaccination records. But in PNG, the so-called baby book is missed very often. In a survey conducted by Samiak, according to the medical records of 70 patients and based on the interview with parents, only $15 \%$ of children had complete vaccination status [11]. In our analysis, only $18 / 56$ had patients had baby books; $68 \%$ had no vaccination records. Based on vaccination records, half of the patients had only one vaccination visit.

The main limitation of the study is a small sample of questioned parents. Taking into consideration local customs, it is challenging to schedule extensive studies in PNG. Indigenous people are very wary of contact with strangers.

There is a shortage of healthcare professionals in PNG, and because of their workload, they are not very eager to be involved with surveys. So this is why we should have accepted this small study as the only possible way of gaining insight into PNG vaccination practices. The small sample can bias our data. We are aware that this may not be representative of the whole population. Parents living in big cities may have different opinions on vaccinations. But the vast majority of people in PNG live in villages, and most of the children are born there.

Second, only written records (vaccination cards) were accepted. It can cause some inaccuracies in vaccination history. It is possible that some more doses were given. But because of a high illiteracy rate, it is not possible to rely on parents' reports.

When it comes to data on vaccination coverage CHS covers with their services around $1 / 4$ of the birth cohort in all the provinces, so the analyzed data are representative for PNG.

Improving vaccination coverage in PNG is essential for outbreak control. To achieve this, it is necessary to improve the quality of services delivered by healthcare facilities and increasing community awareness of the role of vaccinations. As observed in our analysis, declining coverage rates are in accordance with data provided by GAVI, WHO, and Country Official estimates. This is a very worrisome trend.

\section{Conclusion}

The results of this analysis demonstrate that in PNG, the majority of children are not fully immunized. As protection from diseases is low, there is a very high risk of an outbreak of the vaccine-preventable disease in the community.

There are significant differences in vaccination coverage between provinces. This can be improved by a better distribution of healthcare services, especially in rural areas. 
The problem is a lack of public understanding of the need for vaccinations - this can be improved by media campaigns oriented to inform people about the benefits of vaccinations.

Lack of patient vaccination records makes it difficult to establish individual vaccination history.

There is a need for a central registry, where each given dose of vaccine will be recorded.

\section{Supplementary Information}

The online version contains supplementary material available at https://doi. org/10.1186/s12879-021-05824-2.

Additional file 1:. Questionnaire TOKPISIN RURAL. Questionnaire in English and TOKPISIN.

\section{Abbreviations \\ AroB: Autonomous region of Bougainville; BCG: Bacillus calmette-guérin; DTP: Diptheria, tetanus, pertussis; EH: Eastern highlands; ENB: East New Britain; EPI: Expanded Program on Immunization; ES: East sepik; HepB: Hepatitis B; Hib: Haemophilus influenzae type B; IPV: Inactivated poliovirus vaccine; MR: Measles, and rubella; MCH: Maternal and child health clinics; NCD: National capital district; OPV: Oral poliovirus vaccine; PCCLO: Provincial cold chain logistics officers; PNG: Papua New Guinea; SH: Southern Highlands; TB: Tuberculosis; T: Tetanus toxoid vaccine; WH: Western highlands; WHO: World health Organization; WNB: West New} Britain

\section{Acknowledgements}

Not applicable.

\section{Authors' contributions}

EG study design, statistical analysis, data interpretation, manuscript preparation, literature search, JK study design, data collection, manuscript preparation, DJL study design, data interpretation, manuscript preparation, literature search. The author (s) read and approved the final manuscript.

\section{Funding}

Not applicable.

\section{Availability of data and materials}

The datasets used during the current study are available from the corresponding author on reasonable request.

\section{Ethics approval and consent to participate}

An informed verbal consent was obtained from all participants. Being aware of the high illiteracy, the ethics committee approved this procedure. The study received the approval of the ethics committee of the Divine Word University in PNG.

\section{Consent for publication}

NA

\section{Competing interests}

All the authors declare no competing interests.

\section{Author details}

${ }^{1}$ Health Promotion Department, Poznan University of Medical Sciences, Fredry 10, 61-701 Poznan, Poland. ${ }^{2}$ Faculty of Medicine and Health Sciences, Divine Word University, Madang, Papua New Guinea.
Received: 17 June 2020 Accepted: 20 January 2021

Published online: 30 January 2021

\section{References}

1. National Statistical Office of Papua New Guinea. Demographic health survey Available at: https://www.nso.gov.pg/download/81/demographic-healthsurvey/2181/demographic-health-survey-2016-18-report.pdf.

2. Government P. PNG National Census data 2011. http://sddspcint/en/ resources/document-library?view=preview\&format=raw\&fileld=218. 2011.

3. Annual Report on Child Morbidity and Mortality 2017. Child Health Advisory Committee, editor. Port Moresby: PNG National Department of Health; 2017.

4. GAVI Alliance Annual Progress Report 2013. Available at http://www. gavialliance.org/country/PD. ePlatform - APR (gavi.org).

5. United Nations/UNICEF. Papua New Guinea consolidated results report for 2012-2015 Planning. Available from:http://www.unicef.org/about/ execboard/files/Papua_New_Guinea_CRR\%2D\%2D2008-2011_with_ summary.pdf

6. WHO vaccine-preventable diseases: monitoring system. 2020 global summary. Papua New Guinea reported immunization coverage [database on the Internet] http://apps.who.int/immunization_monitoring/ globalsummary/countries?countrycriteria\%5Bcountry\%5D\%5B\%5D=PNG

7. Health PNGNDo. Sector Performance Annual Review: 2011-2015. Accesed online: http://wwwhealthgovpg/publications/2015_SPARpdf. 2015.

8. Morgan C. Enhancing pregnancy outcomes. A joint program of UNICEF PNG and the PNG National Department of Health, implemented by the PNG National Institute of Medical Research: Burnet Institute 2008; 22nd April.

9. Russo G, Miglietta A, Pezzotti P, et al. Vaccine coverage and determinants of incomplete vaccination in children aged 12-23 months in Dschang, west region, Cameroon: a cross-sectional survey during a polio outbreak. BMC Public Health. 2015;15:630.

10. Toikilik S, Tuges G, Lagani J, Wafiware E, Posanai E, Coghlan B, et al. Are hard-to-reach populations being reached with immunization services? Findings from the 2005 Papua New Guinea national immunization coverage survey. Vaccine. 2010;28:4673-9.

11. Samiak L, Emeto TI. Vaccination and nutritional status of children in Karawari, East Sepik Province, Papua New Guinea. PLoS One. 2017;12(11): e0187796.

12. UNICEF Papua New Guinea: Humanitarian Situation Report No. 4 (28 April14 May 2018) - Papua New Guinea | ReliefWeb.int.

13. Wiesen E, Lagani W, Sui G, Arava J, Reza S, Diorditsa S, Lin Y. Assessment of the hepatitis B birth dose vaccination program, Papua New Guinea, 2014. Vaccine. 2016;34:367-72

14. Riddell M, Senn N, Clements CJ, Hobday L, Cowie B, Kurubi J, Kevin A, Siba P, Reeder JC, Morgan C. Rubella control in Papua New Guinea: age-specific immunity informs strategies for introduction of rubella vaccine. Vaccine. 2012;30(52):7506-12

15. Senn N, Riddell M, Omena M, Siba P, Reeder JC, Clements CJ, et al. Measles in Papua New Guinea: an age-specific serological survey. Vaccine. 2010;28: 1819-23.

16. WHO International. Papua New Guinea to vaccinate 700,000 in second round of polio campaign. WHO International; 2018. [cited 2018 Oct 1]. Available from: http://www.wpro.who.int/papuanewguinea/mediacentre/ releases/20180823-png-secondround-polio-vaccination/en/

17. WPRO. Polio immunization drive starts 16 July in Papua New Guinea: WPRO; 2018. [cited 2018 Oct 1]. Available from: http://www.wpro.who.int/ papuanewguinea/mediacentre/releases/20180711/en/

\section{Publisher's Note}

Springer Nature remains neutral with regard to jurisdictional claims in published maps and institutional affiliations. 